\title{
Investigation of Al-ZERODUR interface by Raman and secondary ion mass-spectroscopy
}

\author{
L.I. Berezhinsky, V.P. Maslov, V.V. Tetyorkin and V.A. Yukhymchuk \\ V. Lashkaryov Institute of Semiconductor Physics, NAS of Ukraine, 41, prospect Nauky, 03028 Kyiv, Ukraine \\ Phone: (38 044) 525-5778
}

\begin{abstract}
The interface of ZERODUR ceramics and thin aluminium film was investigated by Raman and secondary ion mass-spectroscopy techniques. Possible chemical reactions at the interface is briefly analyzed and compared with experimental data. Contributions of amorphous and crystalline phases of ZERODUR to Raman spectra are discussed.
\end{abstract}

Keywords: ZERODUR ceramics, Raman spectra, secondary ion mass-spectroscopy.

Manuscript received 19.04.05; accepted for publication 18.05.05.

\section{Introduction}

ZERODUR is glass ceramic widely used in different brunches of industry and science. For example, it has decisive advantages for application in modern LCD lithography. Among the most important advantages of this material are its zero thermal expansion, good processability and very low surface roughness. From the chemical viewpoint, ZERODUR consists of oxides $\mathrm{Li}_{2} \mathrm{O}-\mathrm{SiO}_{2}-\mathrm{Al}_{2} \mathrm{O}_{3}$. It is synthesized at temperatures 700 $1000^{\circ} \mathrm{C}$. In order to stimulate formation of the nucleation center, titanium oxide is added to the starting charge. This ceramics has both crystalline and amorphous phases. The crystalline phase is represented by crystallites of nanometer sizes $(d=50-70 \mathrm{~nm})$. The crystalline phase has a negative linear thermal expansion, while that of the amorphous phase is positive. This results in zero thermal expansion coefficient in a rather wide temperature range.

ZERODUR serves as the mirror substrate material for large-scale telescopes. Currently the world's largest and most powerful telescope, has four main mirrors made of ZERODUR glass ceramic, each with the surface of over 50 square meters and the diameter of 8.2 meters. Very large mirrors and other systems may be composed of many components made of ZERODUR. So, assembling these components is an important engineering task. Different components made of ZERODUR can be jointed together by diffusion welding by using the aluminium film of $100 \mathrm{~nm}$ thickness previously deposited on the surface in a vacuum chamber. The performance of such welding depends critically on chemical and physical processes at the aluminium-ZERODUR ceramics interface. The aim of this work is to investigate these processes at the interface of ZERODUR ceramics with aluminium.

\section{Experimental results and discussion}

Raman spectroscopy is known to be an excellent tool to study local order and its perturbation in both crystalline and amorphous materials. Because of the short-range order is preserved in amorphous material, Raman spectra are expected to be similar for amorphous and crystalline phases of investigated material. However, Raman spectroscopy does not allow to decide whether bond angle or bond length fluctuations are predominant. In the present investigation Raman scattering has been measured on selected samples of ZERODUR ceramics. The Ar laser $(\lambda=514.5 \mathrm{~nm}, P \approx 100 \mathrm{~W})$ was used to excite Raman spectra. Experimental spectra were recorded in the photon counting mode.

As the microstructure of investigated material composed from crystalline and amorphous phases is unknown, modeling the amorphous network is difficult. However, it is clear that interpretation of a measured Raman spectrum of one of these phases is possible if there is information on the vibration spectrum of another phase. Taking this into account, the vibration spectrum of $\mathrm{SiO}_{2}$ film grown on a sapphire substrate was measured first of all. The necessity of such measurements is explained by the fact that the characteristic spectral lines corresponding to chemical bonds of $\mathrm{SiO}_{2}$ should be observed also in ZERODUR. The $\mathrm{SiO}_{2}$ glassy represents the amorphous network from silicon atoms tetrahedrally surrounded by oxygen atoms. The tetrahedrons are bound with each other by means of oxygen atoms, so $\mathrm{Si}-\mathrm{O}-\mathrm{Si}$ bridges with the angle 
between bonds close to $150^{\circ}$ is formed. In other words, in the amorphous network each of $\mathrm{Si}$ atom is surrounded by four oxygen atoms and, in turn, each atom of oxygen is surrounded by two atoms of $\mathrm{Si}$ [1].

In Fig. 1, the vibration peaks with frequencies about 800 and $100 \mathrm{~cm}^{-1}$ are shown. The peak at the frequency $400 \mathrm{~cm}^{-1}$ can be attributed to Si-O rocking vibration of $\mathrm{SiO}_{2}$ tetrahedron, and the peak at $800 \mathrm{~cm}^{-1}$ is connected to vibration of chemical bond bending of Si-O-Si bridge [2]. The intensive narrow peak at $378 \mathrm{~cm}^{-1}$ is caused by radiation of plasma in gas-discharge tube of Ar laser.

Curve 2 in Fig. 1 represents the Raman spectrum of the same film after thermal annealing in nitrogen atmosphere for 15 minutes at the temperature $500{ }^{\circ} \mathrm{C}$. It is seen that weak peak occurs also in the range of frequencies around $490 \mathrm{~cm}^{-1}$. It can be attributed to $\mathrm{Si}-\mathrm{Si}$ bond. It has been shown in [3] that thermal annealing of $\mathrm{SiO}_{2}$ films results in the composition transformation

$2 \mathrm{SiO}_{x} \rightarrow x \mathrm{SiO}_{2}+(2-\mathrm{x}) \mathrm{Si}$.

For example, in those parts of $\mathrm{SiO}_{2}$ film where the stoichiometry is breaked, elementary silicon can arise at high temperatures. As shown in [4], high temperature annealing of $\mathrm{SiO}_{2}$ non-stoichiometric films results in broad-band peak with maximum centered at $490 \mathrm{~cm}^{-1}$, which may be attributed to $\mathrm{Si}-\mathrm{Si}$ bond. This means that clusters of amorphous silicon are formed.

To obtain information about vibrations in $\mathrm{Al}_{2} \mathrm{O}_{3}$, the overall (depolarized) Raman spectrum of sapphire was measured, Fig. 2. As seen, three peaks at 419, 646, and $751 \mathrm{~cm}^{-1}$ are clearly observed in the spectrum. Peak at $378 \mathrm{~cm}^{-1}$ is also observed as well as in the spectrum of $\mathrm{SiO}_{2}$. Since the scattering properties of sapphire is less than the film of $\mathrm{SiO}_{2}$, this band in Fig. 2 has a lower intensity. In the case of amorphous $\mathrm{Al}_{2} \mathrm{O}_{3}$ films, it is possible to suppose that the measured spectrum should correspond qualitatively to that shown in Fig. 2, but the width of the observed peaks may be more considerable.

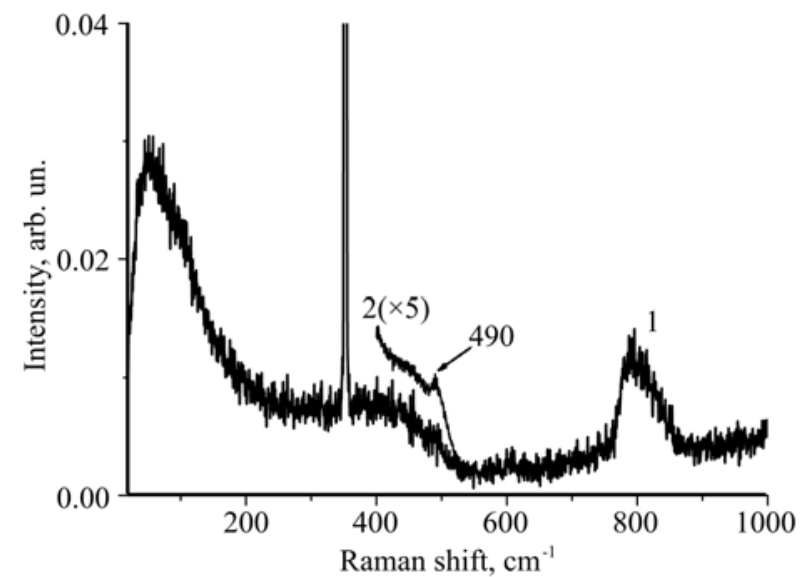

Fig. 1. Raman spectra of the $\mathrm{SiO}_{2}$ amorphous film (curve 1) and the same film annealed at $T=500{ }^{\circ} \mathrm{C}$ for $15 \mathrm{~min}$ (curve 2).

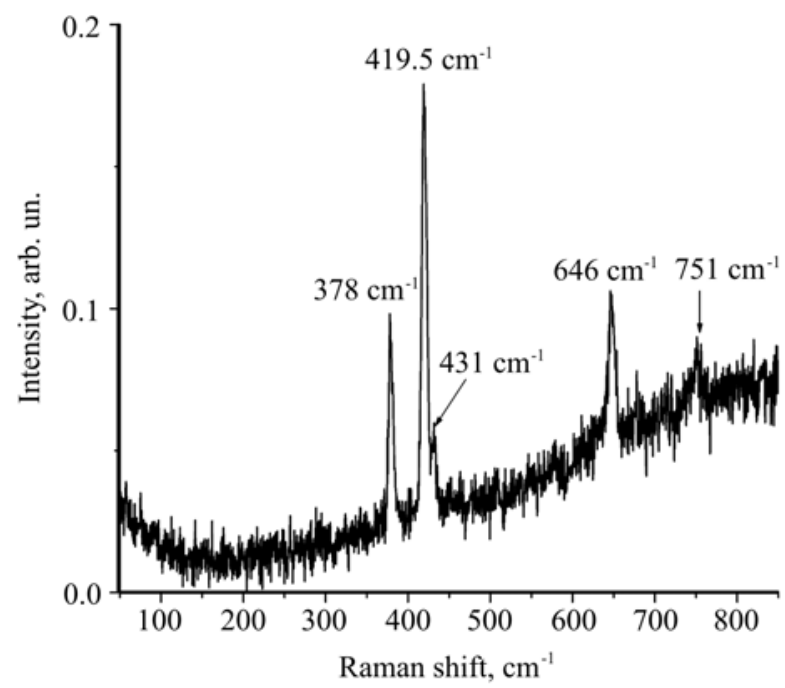

Fig. 2. Raman spectra of sapphire $\left(\alpha-\mathrm{Al}_{2} \mathrm{O}_{3}\right)$.

In order to measure Raman spectra in ZERODUR ceramic samples, the latter were specially prepared. For this purpose, samples were cut in the shape of parallelepiped $15 \times 15 \times 40 \mathrm{~mm}$. Their lateral faces were polished. One of the face was covered by thin film of $\mathrm{Al}$ thermally evaporated in a vacuum system. After that samples were annealed at temperature $400^{\circ} \mathrm{C}$ within 15 minutes. The metallic layer was then removed in such a manner that the trace of $\mathrm{Al}$ was saved in one-half of a sample. The geometry of measurements is shown in Fig. 3.

Fig. 4 shows the Raman spectra measured in several parts of the ZERODUR sample, which are indicated in Fig. 3. The spectra 1 and 2 were recorded using the standard $90^{\circ}$ technique. In the spectrum obtained at bulk excitation of a sample, two broad peaks are observed in two spectral ranges $400-500$ and $100-200 \mathrm{~cm}^{-1}$. The edge of the first peak at frequencies less than $50 \mathrm{~cm}^{-1}$

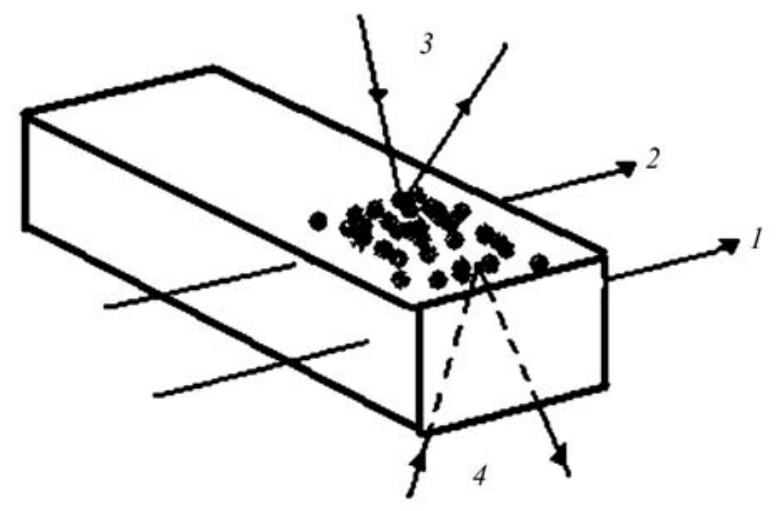

Fig. 3. The geometry of the experiment: 1-4 different positions for Raman spectra detection. 


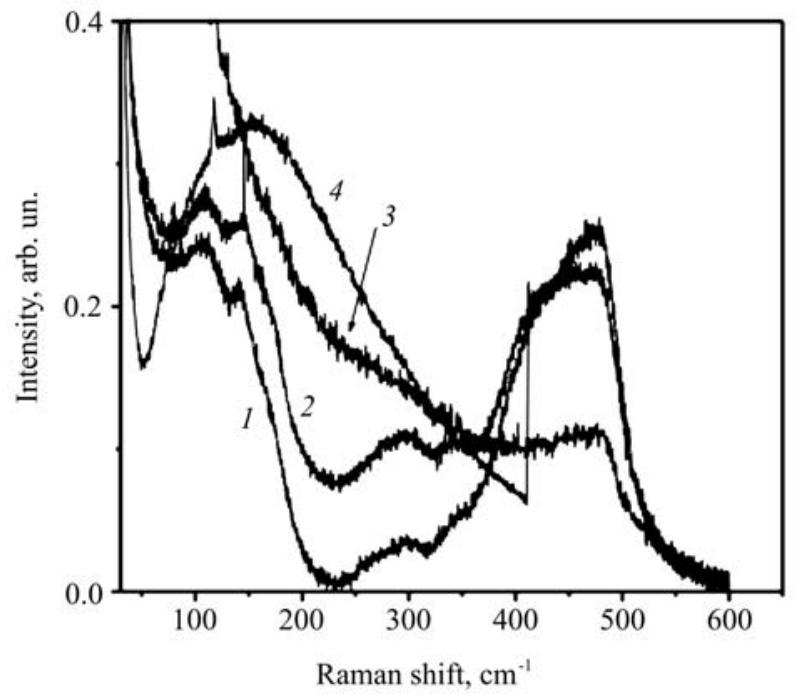

Fig. 4. Raman spectra of ZERODUR: curves 1-4 correspond to positions indicated in Fig. 3.

represents the wing of the Rayleigh line. From the comparison of this spectrum with Raman spectra of $\mathrm{SiO}_{2}$ and $\mathrm{Al}_{2} \mathrm{O}_{3}$ (Figs 1 and 2) one can suppose that the peak in the spectral range $100-200 \mathrm{~cm}^{-1}$ can be attributed to $\mathrm{SiO}_{2}$, whereas that peak in the spectral range 400$500 \mathrm{~cm}^{-1}$ is attributed to $\mathrm{Al}_{2} \mathrm{O}_{3}$. In the crystals of corundum $\gamma-\mathrm{Al}_{2} \mathrm{O}_{3}$ or sapphire- $\mathrm{Al}_{2} \mathrm{O}_{3}$, any aluminium atom occupies position with octahedral surrounding of oxygen atoms. In amorphous phase, it has the same nearest surrounding. However, in the amorphous network little changes of angles and lengths of chemical bonds can occur. This results in changes of vibrations frequencies inherent to the elementary octahedron, and this is a cause of a large broadening of observed spectral peaks.

It is well known that reactivity of $\mathrm{Al}$ is higher as compared to Si. Thus, it may be reasonably assumed that the diffusion of $\mathrm{Al}$ into ZERODUR is accompanied by the chemical reaction

$$
4 \mathrm{Al}+3 \mathrm{SiO}_{2}=2 \mathrm{Al}_{2} \mathrm{O}_{3}+3 \mathrm{Si} .
$$

This transformation is possible, if the heat of reaction (2) is negative. Note that the energy of chemical bonds in $\mathrm{Al}_{2} \mathrm{O}_{3}$ is approximately $20 \%$ higher than in $\mathrm{SiO}_{2}$. This can be regarded as additional proof that exchange reaction can be carried out at $400^{\circ} \mathrm{C}$. However, there arises a problem concerning possible positions of $\mathrm{Al}$ in the investigated ceramics. In the crystal lattice of $\mathrm{SiO}_{2}$, aluminium can occupy both substitutional and interstitial positions. Because of $\mathrm{Al}$ has smaller radius as compared to $\mathrm{Si}$, substitution of $\mathrm{Si}$ by $\mathrm{Al}$ in $\mathrm{SiO}_{2}$ tetrahedral structures will cause a shift of peak frequencies towards higher frequencies. Curve 4 in Fig. 4 represents the Raman spectrum measured at the part of the surface where this process is the most probable. This result qualitatively confirms the above reasons. On the other hand, Al can substitute $\mathrm{Si}$ in amorphous phase, too. In this case, it is possible to expect that clusters of amorphous $\mathrm{Si}$ are formed. The Raman spectrum shown in Fig. 4 (curve 3) was measured from the surface that has been previously covered by Al. The weak peak at $490 \mathrm{~cm}^{-1}$ can be attributed to Si-Si bonds. Furthermore, the behavior of experimental curve in the spectral range $400-500 \mathrm{~cm}^{-1}$ qualitatively coincides with curve 2 in Fig. 1, which corresponds to silicon amorphous clusters in $\mathrm{SiO}_{2}$.

In addition, it is necessary to note that the existence of the alumina $\mathrm{Al}_{2} \mathrm{O}_{3}$ amorphous phase should not be excluded in glass ZERODUR. The structure of the amorphous network is unknown in this case. If chemical bonds in the amorphous phase are occupied, it is possible to suppose that in this case the structural unit should be $\mathrm{AlO}_{3}$. In this case, each atom of $\mathrm{Al}$ is bound with three atoms of oxygen, and each atom of oxygen is bound with two atoms of aluminium through a bridge Al-O-Al. Investigation of Raman spectra in $\mathrm{Pr}_{\mathrm{x}} \mathrm{Nd}_{1-\mathrm{x}} \mathrm{AlO}_{3}$ crystals [5] that contain the $\mathrm{AlO}_{3}$ as structural units showed that $\mathrm{AlO}_{3}$ has vibration frequencies in the spectral ranges $50,150,270$, and $480 \mathrm{~cm}^{-1}$. These frequencies are in good agreement with measured spectra (curve 1 and 2, Fig. 4).

Mechanical properties of joint may depend on chemical processes at the metal-ZERODUR interface which are carried out during their manufacture and storage. Chemical reactions may include interdiffusion of metal and components of ZERODUR, formation of mixture phases and new chemical compounds. Besides, the possible out-diffusion of components $(\mathrm{Si}, \mathrm{Li})$ into the metal can modify its physical properties. Naturally, the diffusion processes at the interface depend on temperature. In this case, temperature is an important parameter not only from the view point of diffusion velocity but the activation energy of possible chemical reactions, too. For example, the exchange reaction (2) seems to be the most simple type of possible chemical reactions at the interface.

In order to clarify physical properties of the Al-ZERODUR interface, the secondary ion massspectroscopy (SIMS) measurements has been carried out. The SIMS profile measurements were taken with IMS-4F ("Cameca"). SIMS profiling was performed with a $4 \mathrm{keV} \mathrm{Ar}^{+}$primary beam scanned over a crater approximately $2 \times 2 \mathrm{~mm}$. The results of SIMS measurement are shown in Fig. 5. The out-diffusion of $\mathrm{Si}$ into the metal film is clearly seen. Moreover, the increase of Si content at the surface of the metal film (the so-called floating of $\mathrm{Si}$ ) is observed as well. This result is in accordance with those of Raman scattering measurements described above. 


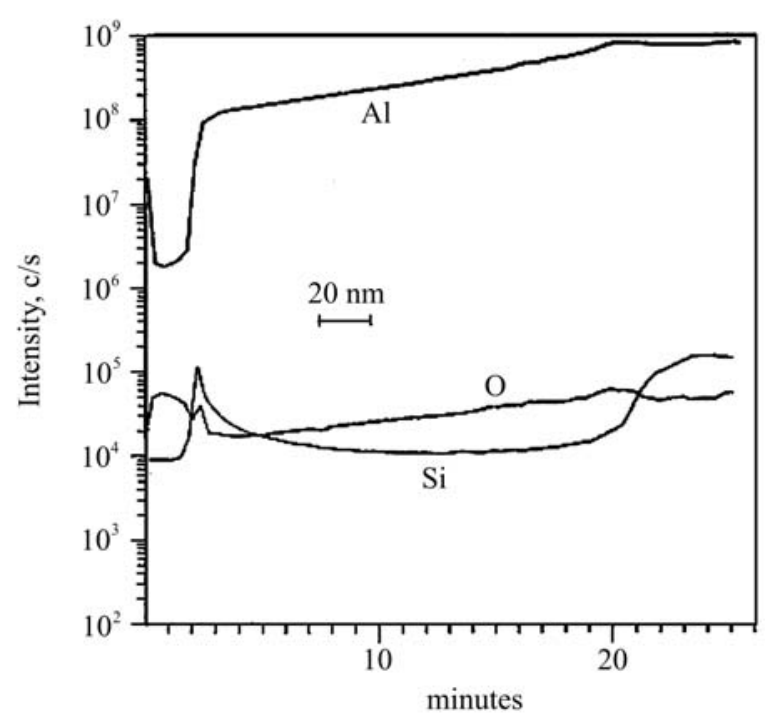

Fig. 5. SIMS profiles of ZERODUR measured from the surface covered by aluminium layer.

\section{Conclusions}

1. Raman spectra are investigated in ZERODUR ceramics at room temperature. Experimental data are analyzed taking into account contributions of amorphous and crystalline phases of ZERODUR to measured spectra.

2. Possible chemical reactions and interdiffusion at the interface of aluminium and ZERODUR ceramics are studied.

\section{References}

1. R. Engelke, Th. Roy, Y.G. Neumann, K. Hubner, Chemical bond and related properties of $\mathrm{SiO}_{2} / /$ Phys. stat. sol.(a) 65, p. 271-280 (1981).

2. A. Lehman, L. Schuma, K. Hubner, Optical phonons in amorphous silicon oxide // Phys. stat. sol. 117, p. 689-697 (1983).

3. F. Rochet, G. Dudour, H. Roulet et. al., Modification of $\mathrm{SiO}$ through room temperature plasma treatments, rapid thermal annealings and laser irradiation in a nonoxidizing atmosphere // Phys. Rev. B 37, p. 6468-6474 (1988).

4. V.L. Bratus', V.A. Yukhymchuk, L.I. Berezhinsky et. al., Structural transformations and creation of $\mathrm{Si}$ nanocrystals in the $\mathrm{SiO}_{\mathrm{x}}$ films // Fiz. Tekhn. Poluprovodn. 35, p. 854-859 (2001) (in Russian).

5. E. Finkman, E. Cohen, L.G. Uittert, Vibrational and electronic Raman scattering in the various phases of $\mathrm{Pr}_{\mathrm{x}} \mathrm{Nd}_{1-\mathrm{x}} \mathrm{AlO}_{3}$. In: Light scattering in solids. Proc. Intern. Conf., Paris, p. 369-372 (1971). 